\title{
Digitally-Assisted Linearization of Wideband Direct Conversion Receivers
}

\author{
${ }^{1}$ Edward A. Keehr, ${ }^{1}$ Ali Hajimiri \\ ${ }^{1}$ Department of Electrical Engineering, California Institute of Technology \\ 1200 E. California Blvd. M/C 136-93, Pasadena, CA 91125, United States of America \\ ${ }^{1}$ keehr@caltech.edu \\ 'hajimiri@caltech.edu
}

\begin{abstract}
A SAW-less direct-conversion receiver is presented which utilizes a mixed-signal feedforward path to regenerate and equalize IM3 products, thus accomplishing system-level linearization. The receiver system performance is dominated by a custom integrated front end realized in $130 \mathrm{~nm}$ CMOS and achieves an uncorrected out-of-band IIP3 of $-7.1 \mathrm{dBm}$ under the worst-case UMTS FDD Region 1 blocking specifications. IM3 equalization results in an effective IIP3 of $+5.3 \mathrm{dBm}$ and reduces total input-referred error by over $23 \mathrm{~dB}$.
\end{abstract}

\section{INTRODUCTION}

Improving the linearity of RF receivers has long been a challenging circuit design problem. As supply voltages drop with process scaling and as market demands continue to press for the elimination of off-chip components, such as SAW filters, standard techniques for meeting receiver linearity specifications will become inadequate. Direct conversion receivers are of considerable interest in this regard, as they constitute the dominant architecture in modern communication systems. Much progress has recently been made in improving the IIP2 of such circuits; however, a great need still exists to improve upon the IIP3 performance. This paper discusses the concept and details behind a system-level approach to satisfy this need.

\section{SySTEM-LEVEL CONCEPTS}

\section{A. System-Level Linearization via Equalization}

In order to improve the system IIP3 for a SAW-less directconversion receiver, the original main path of the receiver is augmented with an alternate receiver path, as shown in Fig. 1 below.

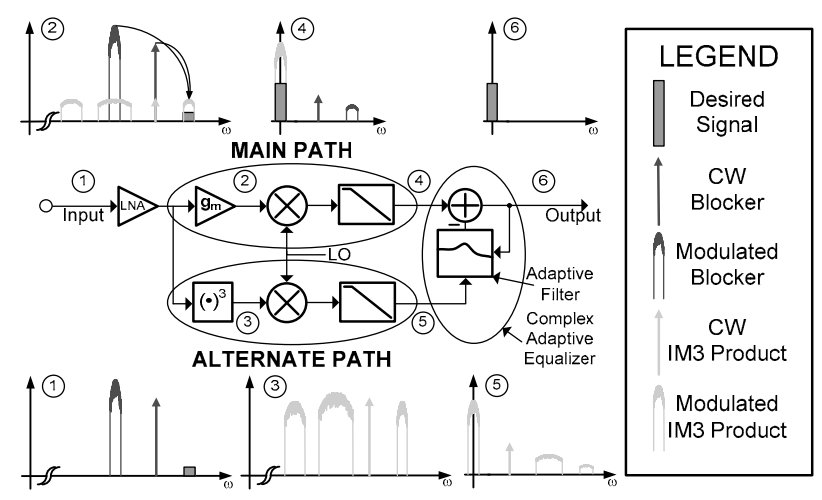

Fig. 1 IM3 equalization concept.
The alternate path generates IM3 products in the analog domain at RF and downconverts them to baseband using the same LO frequency as the main path receiver. The IM3 products are then digitized and are used as inputs to an equalizer which cancels the baseband IM3 products in the main path. Hence, a linearization via equalization is accomplished. Generating the IM3 products after the LNA at $\mathrm{RF}$ is a crucial feature of this architecture, since it is at this point in the system that the blocker magnitudes are at their largest.

This solution has the advantages of being both powerefficient and robust. Since the alternate path must pass only IM3 products, the dynamic ranges of its constituent blocks can be over $10 \mathrm{~dB}$ less than in the main path, allowing for significant power savings in its overall design. As problematic blocker conditions occur roughly less than $10 \%$ of the time, the time-averaged power dissipation of the alternate path is further reduced by powering it on only when needed. The adaptive nature of the equalization guarantees robustness in the presence of changes in temperature, LO frequency, and blocker characteristics.

The idea of adaptively cancelling IM3 products has recently been described in a system-level study [1], where the alternate path resides completely within digital baseband. For IM3 cancellation to occur in this scheme, the ADCs must digitize all possible IM3-producing blockers. For example, in FDD UMTS Region 1 this requires digitizing frequency bands from $1670-1850 \mathrm{MHz}, 2015-2075 \mathrm{MHz}$, and $1920-1980 \mathrm{MHz}$ (TX band), rendering this scheme unattractive from a power efficiency perspective. In order to overcome this issue, the mixed-signal approach proposed in this work passes only the problematic IM3 products through the alternate path ADCs. Hence, the alternate path ADC and digital baseband sampling rate requirements are no greater than those of the original main path.

\section{B. Receiver System Architecture}

The receiver system architecture described in this paper is shown below in Fig. 2. In order to provide a quantitative design objective, the design targets the FDD UMTS Region 1 standard, as it is well known for its linearity challenges. It contains a custom-designed integrated front-end in $130 \mathrm{~nm}$ CMOS, analog baseband circuitry on PCB, and a digital back end implemented on an FPGA platform. 


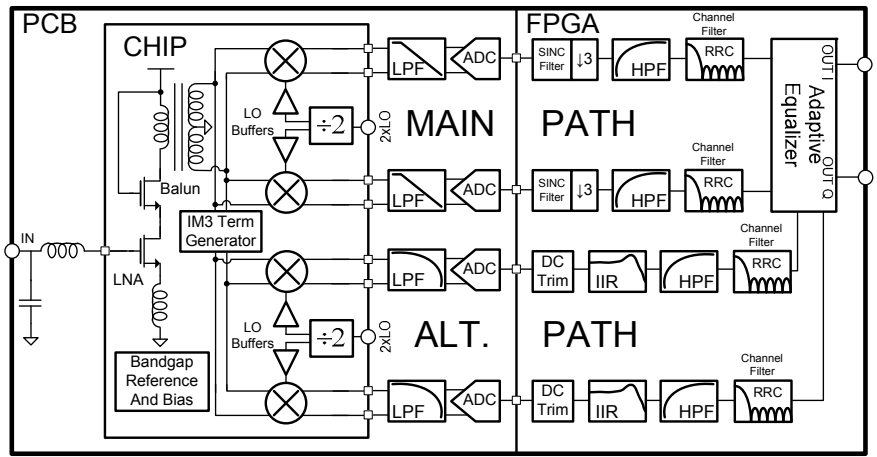

Fig. 2 Experimental UMTS receiver architecture.

Although it is possible to perform the adaptive equalization in analog circuitry, shifting as much of the signal processing burden as possible to the digital domain affords several advantages. For example, the behaviour of digital circuitry is relatively insensitive to process variations, and the continued scaling of digital processes has made baseband digital blocks power-competitive with equivalent blocks in the analog domain.

Considerations specific to modern cellular receivers also factor into the choice of predominantly digital equalization. For example, such receivers often implement adjacent channel rejection filters in the digital domain. To ensure that large adjacent channel signals do not interfere in the adaptive equalization, it is best to place the adaptive equalizer after these filters in the digital domain as well.

\section{ANALOG CIRCUIT DESCRIPTIONS}

\section{A. Main Path Circuitry}

As shown in Fig. 2, the LNA is an inductively degenerated cascode architecture which is terminated by a transformer balun. This balun serves as the LNA load inductor and subsumes the single-ended-to-differential conversion previously handled by the interstage SAW filter. The balun is followed by high-IIP2 mixers [2] which are folded in order to function under the $1.2 \mathrm{~V}$ supply voltage. This choice obviates any IM2 equalization and allows the design effort to focus solely on IIP3 improvement. A frequency divide-by-two circuit which generates I and Q LO phases is also included on chip in order to minimize LO coupling into the substrate and chip ESD guard ring.

The main path baseband circuitry is implemented with discrete, commercially available components. The baseband low-pass filter is a third order Chebyshev architecture and is followed by an 8 -bit pipelined ADC running at $50 \mathrm{MHz}$.

\section{B. Alternate Path Circuitry}

The alternate path is a low-power, low-area variant of the main path, with the primary difference being the inclusion of an IM3 term generator. In order to conserve area, the alternate path mixer dispenses with the tuning inductor present in the main path mixer. Another I/Q generating frequency divider is used for the alternate path, as routing LO signal from the main path frequency divider increases the risk of LO coupling to other blocks in the system.
Like the main path, the alternate path baseband circuitry is implemented with discrete components. The alternate path baseband low-pass filter is a single real-pole embedded into the ADC buffer, which drives an 8-bit pipelined ADC running at $16.66 \mathrm{MHz}$. Along with the free real pole at the output of the mixer, this level of postfiltering is sufficient to attenuate any IM3 products not involved in baseband equalization to below the alternate path noise floor.

\section{IM3 TERM GENERATOR}

The IM3 term generator is an unconventional block, and as such, it merits additional consideration in its design. A pure cubing circuit is desired, as it passes negligible linear terms. If present, these terms will be treated as error by the adaptive equalizer, potentially degrading the receiver small signal gain, IM3 correction ratio, or both. Unlike other approaches that utilize the third-order Taylor series coefficient of the MOSFET [3], the design concept used for this IM3 term generator, shown below in Fig. 3, exploits the stronger second-order term by distributing the cubing between a conventional MOS squaring circuit and a Gilbert multiplier. The circuit schematic itself is shown in Fig. 4.

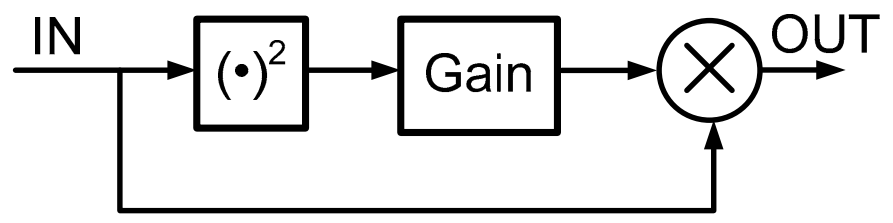

Fig. 3 Multistage cubing concept.

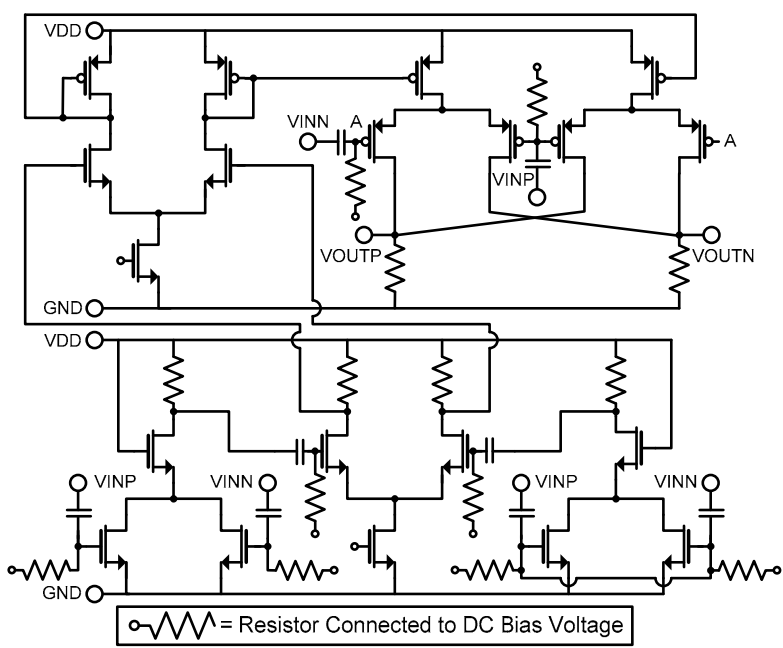

Fig. 4 IM3 term generator schematic.

As it produces a single-ended output, the squaring circuit must be followed by an active balun. To improve the generator CMRR, the negative terminal of the balun is tied to a replica squaring circuit whose gate terminals are shorted. This branch only generates common mode signal, which is then rejected by the CMRR of the balun.

Perhaps the most important aspect of the proposed IM3 term generator is the multistage nature of the cubing. Only the beat frequency terms of the squaring are required to be retained in order to complete the cubing operation. Hence, the 
bandwidth of the inter-multiplication circuitry can be substantially smaller than the RF frequencies of the blocker signals, as depicted in Fig. 5 below. In this case, the gainbandwidth principle can be used to the designer's advantage, as substantial gain can be applied for less power than if the full IM2 spectrum up to $4 \mathrm{GHz}$ were retained in between nonlinear operations.

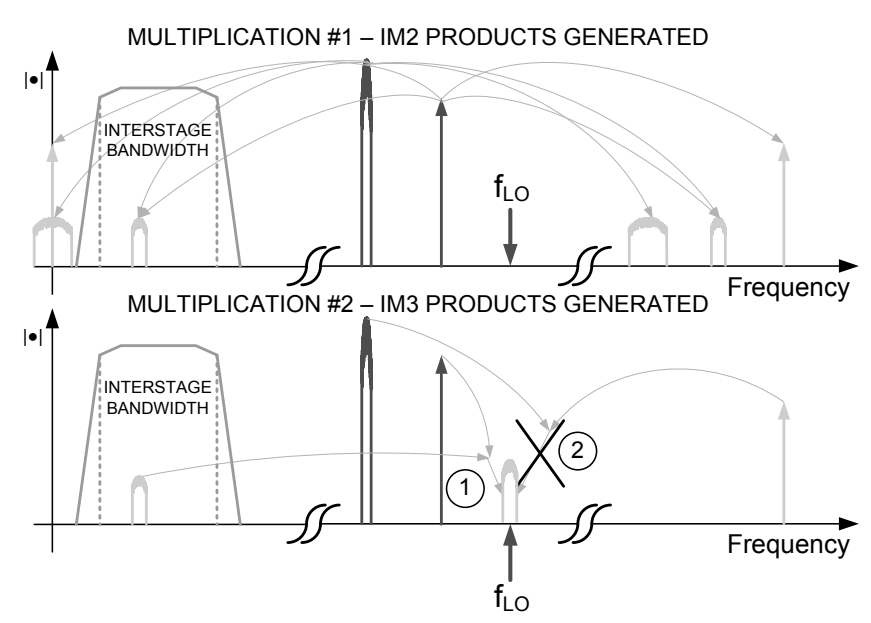

Fig. 5 Multistage cubing: frequency domain considerations.

\section{Digital EQUaLIZATION}

The path equalization implemented in this project is performed in the digital domain and is partitioned into fixed and adaptive portions. This choice stems from the fact that the minimal analog postfilters of the main and alternate path were found to be different in both type and order. Adaptive equalization of such a known IIR path difference is computationally inefficient. Therefore, the fixed equalization consists of a three-multiplier IIR filter in the alternate path. The remaining difference between the two paths is a complex DC gain and a small random mismatch in the baseband transfer function. This difference is broadband in the frequency domain and by the duality principle corresponds to a small number of FIR taps required in the adaptive equalizer.

The normalized LMS (NLMS) algorithm was chosen for the adaptive equalization scheme due to its simplicity and convergence speed. The division associated with this algorithm can be log2-quantized, allowing the use of a barrel shifter as a divider. Although a complex LMS-based algorithm such as NLMS can equalize the phase skew between the main and alternate paths, in general the presence of I-Q mismatch on either path limits the available IM3 cancellation. As shown in Fig. 6 below, an additional degree of freedom in the design was added to overcome this issue by feeding back the complex corrected signal back to independent I and Q taps on each of the incoming alternate path signals.

Yet another consideration is that the performance of the adaptive equalizer is limited in the presence of DC offset. To solve this problem, the proposed design includes high-pass filters in the digital domain and DC offset trimming circuitry in the alternate path. Periodic trimming must be performed prior to the alternate path high-pass filters, or the step response incurred when enabling the digital portion of the alternate path will result in an exponential error transient at the output, prolonging equalizer convergence.

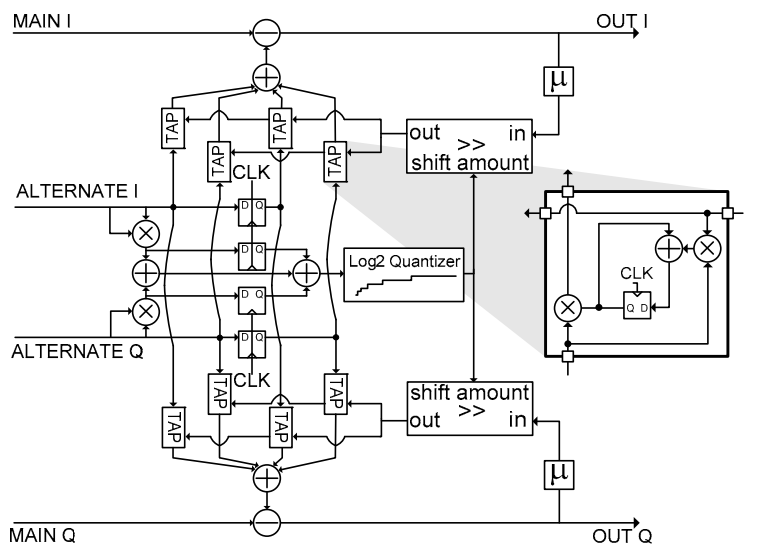

Fig. 6 Enhanced NLMS adaptive equalizer block diagram.

\section{MeAsurement ANd PerformanCe}

The emphasis of this project is to meet the IIP3 requirements implicitly posed by the UMTS out-of-band blocking test, which must be performed while the TX path is operating at maximum output power [4]. For the duplexer [5], the worst case specified IMD condition, with values referred to the LNA input, is $-26 \mathrm{dBm}$ TX leakage at $1.98 \mathrm{GHz}$, a $-34 \mathrm{dBm} \mathrm{CW}$ blocker at $2.05 \mathrm{GHz}$, when the receiver LO frequency is set to $2.12 \mathrm{GHz}$. Therefore, under experiment, the receiver is subject to a modified two-tone test, where one of the signals is QPSK-modulated and set to UMTS standards. Accounting for the $2 \mathrm{~dB}$ loss of the duplexer and the $3 \mathrm{~dB}$ increase in noise margin allowed under blocking conditions, the maximum allowed total input-referred error is $-98 \mathrm{dBm}[6]$. The results of this test are shown in Fig. 7 below and show that in this case under equalization, the input-referred error is $-101 \mathrm{dBm}$. (note: here $\mathrm{f}_{\mathrm{CW}}=2.05125 \mathrm{GHz}, \mathrm{f}_{\mathrm{LO}}=2.1225 \mathrm{GHz}$ ) Also shown in Fig. 7 are the results of the same test using the canonical NLMS algorithm. A main path quadrature mismatch of about $3^{\circ}$ is partially responsible for the higher inputreferred IM3 products.

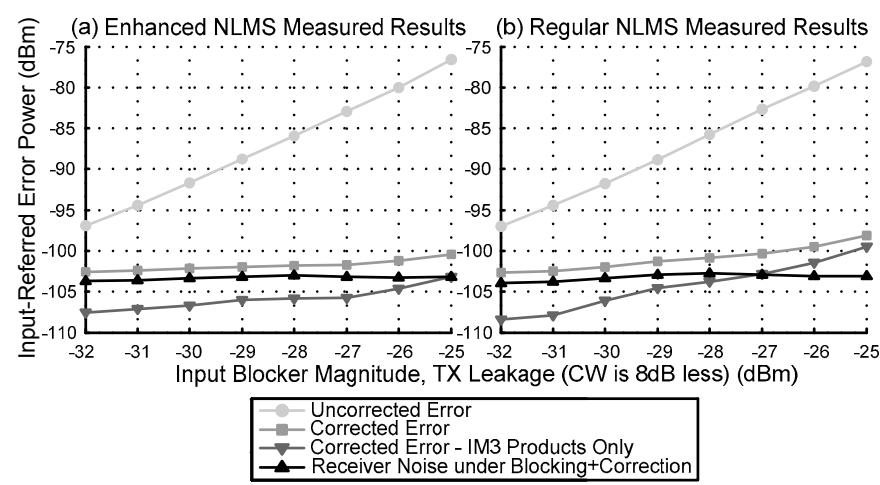

Fig. 7 Measured modified two-tone performance of the receiver, swept over amplitude. 
The total input-referred error accounts for gain loss, thermal noise, IM2, and other IM products. Removing the effect of main path thermal noise and IM2 products yields a lumped input-referred quantity consisting of all other error sources. From this quantity, which is treated as residual IM3 error, a slope-of-3 line is extrapolated from the worst-case input blocker magnitude to obtain an effective IIP3 of $+5.3 \mathrm{dBm}$. This is an improvement of $12.4 \mathrm{~dB}$ from the uncorrected IIP3 of $-7.1 \mathrm{dBm}$. This test was also performed at all 12 UMTS RX frequencies, with the results of this experiment shown below in Fig. 8. Note that in Figs. 7 and 8, the calculated thermal noise of the $50 \Omega$ input impedance is removed to isolate the performance of the receiver circuitry.

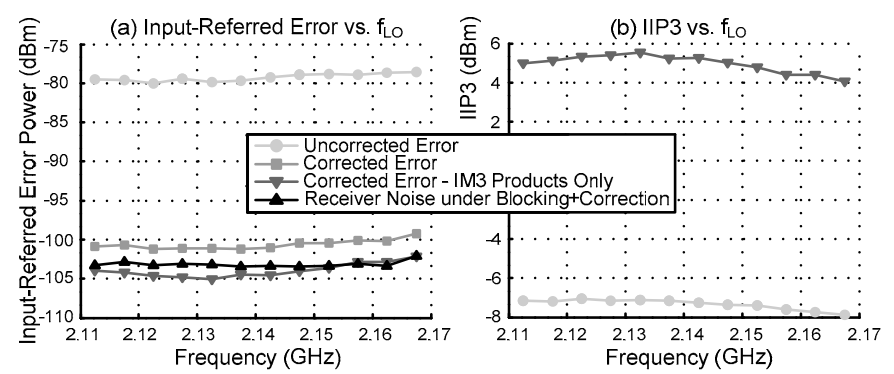

Fig. 8 Measured modified two-tone performance of the receiver, swept over LO frequency. At LNA input TX power is $-26 \mathrm{dBm}, \mathrm{CW}$ power is $-34 \mathrm{dBm}$.

Convergence behavior of the adaptive equalizer is shown in Fig. 9 below for the case where the TX leakage amplitude is $-25 \mathrm{dBm}$ and the $\mathrm{CW}$ blocker amplitude is $-33 \mathrm{dBm}$ at the LNA input at $\mathrm{f}_{\mathrm{LO}}=2.1225 \mathrm{GHz}$.

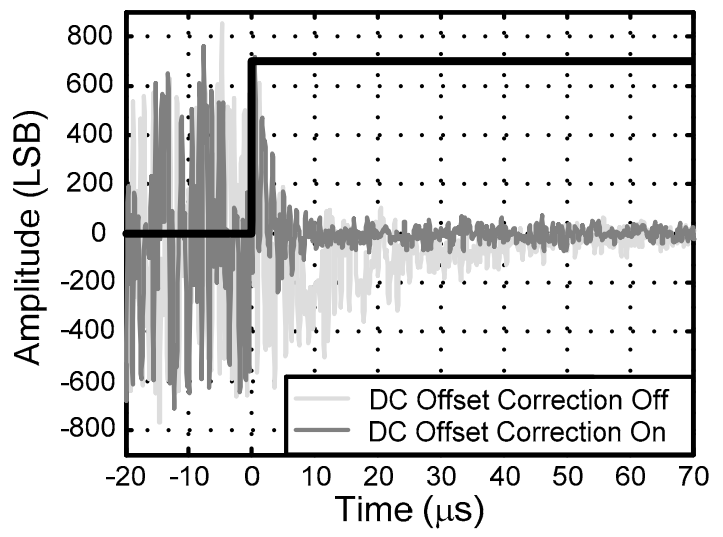

Fig. 9 Measured convergence of the adaptive equalization algorithm.

The measured performance summary of the receiver is shown below in Fig. 10. Fig. 11 shows a die photograph of the integrated RF CMOS front end. Digital power and area numbers are pre-layout estimates derived from a 90nm CMOS standard cell library with estimated wiring parasitics.

\section{CONCLUSION}

This paper describes a UMTS receiver with an integrated front end that cancels IM3 products using a novel mixedsignal feedforward loop. Issues regarding the generation of reference IM3 products, path I-Q mismatches, and DC offset are considered, shown to be relevant, and overcome.

\begin{tabular}{|c|c|}
\hline Parameter Measured at $\mathbf{f}_{\mathrm{LO}}=\mathbf{2 . 1 2 2 5 G H z}$ & Result \\
\hline Total Active Analog Die Area & $1.6 \mathrm{~mm} \times 1.5 \mathrm{~mm}$ \\
\hline Active Alternate Path Analog Die Area & $0.5 \mathrm{~mm} \times 0.4 \mathrm{~mm}$ \\
\hline Analog Die Technology Node & $130 \mathrm{~nm}$ CMOS \\
\hline Estimated Digital Alternate Path Area & $0.42 \mathrm{~mm} \times 0.42 \mathrm{~mm}$ \\
\hline Digital Die Technology Node & $90 \mathrm{~nm}$ CMOS \\
\hline Analog Die LNA+Main Path DC Gain & $30.5 \mathrm{~dB}$ \\
\hline Complete LNA+Main Path DC Gain to ADC Input & $70.2 \mathrm{~dB}$ \\
\hline Return Loss (S11) 2.11GHz-2.17GHz & $<-13 \mathrm{~dB}$ \\
\hline IIP2@1.98GHz & $+58 \mathrm{dBm}$ \\
\hline Uncorrected IIP3 @1.98GHz/2.05125GHz & $-7.1 \mathrm{dBm}$ \\
\hline Effective IIP3@1.98GHz/2.05125GHz & $+5.3 \mathrm{dBm}$ \\
\hline ICP1@1.98GHz & $-19 \mathrm{dBm}$ \\
\hline Analog Die LNA+Main Path NF & $5.1 \mathrm{~dB}$ \\
\hline Complete LNA+Main Path NF & $5.5 \mathrm{~dB}$ \\
\hline Analog Die Supply Voltage & $1.2 \mathrm{~V} / 2.7 \mathrm{~V}$ \\
\hline Analog Die LNA+Main Path Current & $28 \mathrm{~mA}(1.2 \mathrm{~V})$ \\
\hline Analog Die Alternate Path Current & $6.7 \mathrm{~mA}(1.2 \mathrm{~V})$ \\
\hline Estimated Digital Alternate Path Current & $5.6 \mathrm{~mA} \mathrm{(1.0V)}$ \\
\hline Baseband Signal Measurement Bandwidth & $10 \mathrm{kHz}-1.92 \mathrm{MHz}$ \\
\hline
\end{tabular}

Fig. 10 Measured performance summary of receiver.

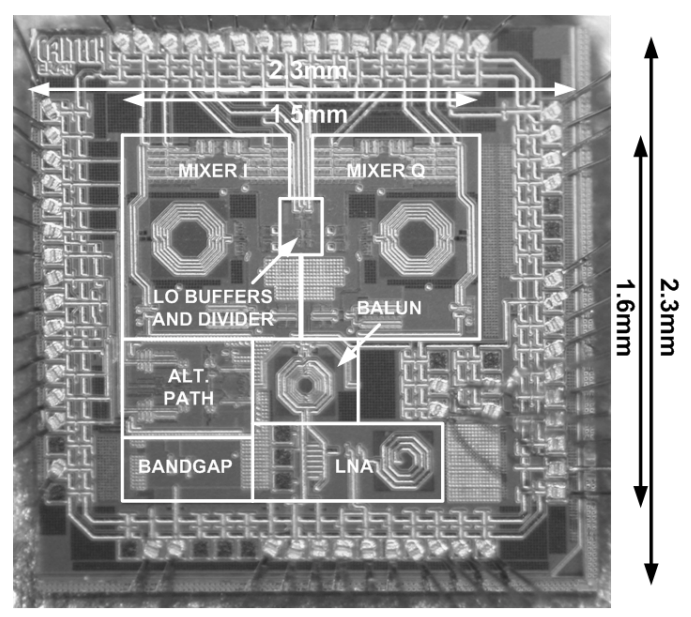

Fig. 11 RF CMOS front-end analog die.

\section{ACKNOWLEDGEMENTS}

The authors thank F. Bohn for the frequency divider IP and H. Mani and J. Yoo for testing assistance. This project was supported by an NDSEG fellowship and the Lee Center for Advanced Networking.

\section{REFERENCES}

[1] M. Valkama, A.S.H. Ghadam, L. Antilla and M. Renfors, "Advanced digital signal processing techniques for compensation of nonlinear distortion in wideband multicarrier radio receivers," IEEE Trans. Microwave Theory and Techniques, vol. 54, pp. 2356-66 June 2006.

[2] A. Liscidini, M. Brandolini, D. Sanzogni and R. Castello, "A $0.13 \mathrm{~mm}$ CMOS front-end, for DCS1800/UMTS/802.11b-g with multiband positive feedback low-noise amplifier," IEEE Journal Solid State Circuits, vol. 41, pp. 981-9, Apr. 2006.

[3] F. Shearer and L. MacEachern, "A Precision CMOS Analog Cubing Circuit," IEEE NEWCAS, pp. 281-284, June 2004.

[4] "UE Radio Transmission and Reception (FDD)," Tech. Specification Group, 3GPP, (TSG) RAN WG4, TS 25.101, v8.1.0, December 2007.

[5] muRata Corp Part Number DFYK61G95LBJCA, http://www.murata.com. Data sheet available at http://smartdata.usbid.com/datasheets/usbid/dsid/103495.pdf.

[6] A. Springer, L. Maurer and R. Weigel, "RF system concepts for highly integrated RFICs for W-CDMA mobile radio terminals," IEEE Trans. Microwave Theory and Techniques, vol. 50, pp. 254-267, Jan. 2002. 